\title{
Dos métodos de fertilización en el cultivo de piña (Ananas sativus) hibrido MD2
}

Two methods of fertilization in the cultivation of hybrid pineapple (Ananas sativus) MD2

\section{Dois métodos de fertilização no cultivo de piña (Ananas sativus) híbrido MD2}

\author{
Urrea González Leidy Alejandra ${ }^{1}$, Ospina Rodrígez Yeisson Esmith ${ }^{1}$ y \\ Bastidas López Harold² \\ ${ }^{1} \mathrm{IA}$, Universidad de los Llanos y ${ }^{2}$ IA, MSc, Docente Universidad de los Llanos \\ hbastidas@unillanos.edu.co
}

Recibido 22 de Noviembre 2017, Aceptado 30 de Abril 2018

\section{RESUMEN}

El cultivo de piña tiene una alta producción en Latinoamérica y ha incrementado su producción en los últimos años en países como Uruguay, Brasil, Ecuador, Costa Rica y Colombia, de la mano de procesos de desarrollo social, lo que ha logrado beneficiar a muchos agricultores. Este experimento se realizó en San Martin, Meta, Colombia, donde fue sembrada la piña variedad MD2 en distancias de 30 $\mathrm{cm}$ entre plantas por $40 \mathrm{~cm}$ entre líneas en cama y de un metro entre camas; se realizó evaluación de dos metodologías de aplicación: T1 = fertilización dirigida al suelo, T2 = fertilizante aplicado en la axila de la planta, ambos casos con dosis exacta; y se compararon con un testigo (T3) en donde no se aplicó ningún fertilizante. En este proyecto se estableció un diseño en bloques completos al azar, con tres tratamientos y cinco repeticiones, cada repetición estuvo constituida por 120 plantas por cama (600 plantas por tratamiento), que correspondió a una unidad experimental, evaluando cada 15 días (15, 30. 45 y 60 días) cinco plantas/cama/repetición, bajo un análisis de varianza con prueba de comparación de Duncan. A los 15 y 30 días de evaluación T1 y T2 presentaron las mayores de alturas de la planta $(P<0.05)$ con valores de 15.18 y 43.60 y 16.24 y $53.20 \mathrm{~cm}$ respectivamente, en comparación con el testigo T3 (13.93 y $39.44 \mathrm{~cm})$. A los 45 y 60 días T2 mostró una altura superior $(\mathrm{P}<0.05)$ con valores de 77.90 y $94.66 \mathrm{~cm}$ 
en comparación con T1 66.52 y 88.30; y T3 68.26 y 79.90 cm respectivamente; aunque a los 45 días T1 y T3 mostraron un comportamiento similar $(P>0.05)$. Según los métodos de fertilización evaluados, el de aplicación directa al suelo evidenció mejor respuesta por parte de la planta en su sistema radicular y foliar, en comparación con los tratamientos de fertilización axilar y sin aplicación de productos (testigo), puesto que las plantas sometidas a fertilización directa en el suelo (T2) presentaron características de desarrollo más eficientes para la absorción de nutrientes.

Palabras clave: Fertilizante, fruta, sistema radicular, hojas.

\section{ABSTRACT}

Pineapple cultivation has a high production in Latin America and has increased its production in recent years in countries such as Uruguay, Brazil, Ecuador, Costa Rica and Colombia, from the hand of processes of social development, which has benefited many farmers. This experiment was carried out in San Martin, Meta, Colombia, where the pineapple variety MD2 was planted at distances of $30 \mathrm{~cm}$ between plants by $40 \mathrm{~cm}$ between lines in bed and one meter between beds; evaluation of two application methodologies was carried out: both cases with exact dose; and were compared with a control (T3) where no fertilizer was applied. In this project a randomized complete block design was established, with three treatments and five repetitions, each repetition was constituted by 120 plants per bed ( 600 plants per treatment), which corresponded to an experimental unit, evaluating every 15 days $(15,30.45$ and 60 days) five plants/bed/repetition, under an analysis of variance with Duncan's comparison test. At 15 and 30 days of evaluation $T 1$ and $T 2$ presented the highest plant heights $(P<0.05)$ with values of 15.18 and 43.60 . and 16.24 and $53.20 \mathrm{~cm}$ respectively, in comparison with the control T3 (13.93 and $39.44 \mathrm{~cm}$ ). At 45 and 60 days T2 showed a higher height $(\mathrm{P}<0.05)$ with values of 77.90 and $94.66 \mathrm{~cm}$ compared with $\mathrm{T} 166.52$ and 88.30; and T3 68.26 and $79.90 \mathrm{~cm}$ respectively; although at 45 days $\mathrm{T} 1$ and T3 showed a similar behavior $(P>0.05)$. According to the fertilization methods evaluated, the direct application to the soil showed better response by the plant in its root and leaf 
system, compared with axillary fertilization treatments and without application of products (control), because the plants subjected to direct fertilization in the soil (T2) presented more efficient development characteristics for the absorption of nutrients.

Keywords: Fertilizer, fruit, root system, leaves.

\section{RESUMO}

O cultivo de piña tem uma alta produção na América Latina e aumentou sua produção nos últimos anos em países como Uruguai, Brasil, Equador, Costa Rica e Colômbia, da mao de processos de desenvolvimento social, que beneficiaram muitos agricultores. Este experimento foi realizado em San Martin, Meta, Colômbia, onde a variedade de piña MD2 foi plantada a distâncias de $30 \mathrm{~cm}$ entre plantas por $40 \mathrm{~cm}$ entre linhas no leito e um metro entre leitos; avaliação de duas metodologias de aplicação: ambos os casos com dose exata; e foram comparados com um controle (T3) onde nenhum fertilizante foi aplicado. Neste projeto foi estabelecido um delineamento em blocos ao acaso, com três tratamentos e cinco repetições, cada repetição consistiu de 120 plantas por cama (600 plantas por tratamento), o que correspondeu a uma unidade experimental, avaliando cada 15 dias (15, 30. 45 e 60 dias) cinco plantas/cama/repetição, sob uma análise de variância com o teste de comparação de Duncan. Aos 15 e 30 dias de avaliação T1 e T2 apresentaram as maiores alturas de plantas $(P<0.05)$ com valores de 15.18 e 43.60 e e 16.24 e $53.20 \mathrm{~cm}$, respectivamente, em comparação com o controle T3 (13.93 e $39.44 \mathrm{~cm}$ ). Aos 45 e 60 dias, T2 apresentou maior estatura $(\mathrm{P}<0.05)$, com valores de 77.90 e $94.66 \mathrm{~cm}$, comparados aos T1 66.52 e 88.30; e T3 68.26 e $79.90 \mathrm{~cm}$ respectivamente; embora aos 45 dias T1 e T3 mostraram um comportamento semelhante $(P>0.05)$. De acordo com os métodos de fertilização avaliados, a aplicação direta ao solo mostrou melhor resposta da planta em seu sistema radicular e foliar, em comparação com tratamentos de fertilização axilar e sem aplicação de produtos (controle), porque as plantas submetidas à adubação direta no solo (T2) apresentaram características de desenvolvimento mais eficientes para a absorção de nutrientes. 
Palavras-chave: Adubo, frutas, sistema radicular, folhas.

\section{INTRODUCCIÓN}

La piña (Ananas sativus (Lindl) Schult), es un cultivo propio de países de la zona tropical, esta fruta que proporciona beneficios porque es conocida por sus propiedades diuréticas y su acción en la limpieza del tracto digestivo, como fruta es consumida en fresco y en bebidas preparadas en conserva sola o en conjunto, también se utiliza como postre y como ingrediente dulce en ciertos preparados de la comida oriental (García y Rodríguez, 2011; Aragón et al., 2012); el cultivo tiene una alta producción en Latinoamérica y se ha incrementado su producción en los últimos años en países como Uruguay, Brasil, Ecuador, Costa Rica y Colombia, de la mano de procesos de desarrollo social, lo que ha logrado beneficiar a muchos agricultores. Por características de calidad y sabor la piña es altamente demandante en el país y por sus exigencias como cultivo, son pocas las zonas que pueden y brindarle las condiciones adecuadas para su óptimo desarrollo (Maglianesi, 2013; Gratani, 2014).

El cultivo de piña se considera semi-perenne, desde el punto de vista comercial lo recomendable son hasta dos cosechas por ciclo productivo, siendo la primera cosecha a los 12 meses y una segunda de los 26 o 30 meses de edad. La piña es de la familia Bromeliaceae, es una planta vivaz con una base formada por la unión compacta de varias hojas formando una roseta, de las axilas de las hojas pueden surgir retoños con pequeñas rosetas básales, que facilitan su reproducción vegetativa, las flores dan fruto sin necesidad de fecundación y del ovario hipógino que desarrolla unos frutos en forma de baya, que conjuntamente con el eje de la inflorescencia y las brácteas, dan lugar a una infrutescencia carnosa (sincarpio) (Batagin et al., 2009; MAG, 2010). El fruto maduro se obtiene 135 días después la floración que se prolonga de 30 a 70 días, del tallo central brotan los diferentes tipos de materiales que se pueden utilizar para propagar la piña, como por ejemplo, las hojas se forman en la base del fruto, hijuelos de tallo que se desarrollan en las yemas axilares y retoños que se desarrollaron en el tallo (Acosta, 2008). 
La piña necesita un suelo fértil para poder crecer y producir el fruto, pero si el objetivo principal de los productores es exportar, se deben mantener niveles nutricionales acordes a la calidad que exige el mercado internacional. A partir de ese nivel hay que aplicar fertilizantes para suplir el nutriente faltante que la planta necesita para producir el fruto esperado; como medidas de apoyo para manejar la nutrición de la piña, se deben realizar análisis foliares y de suelo para medir la cantidad de nutrientes que requiere la plantación. El nitrógeno y el potasio son los nutrimentos más importantes para la piña, el primero influye sobre el rendimiento y el segundo sobre la calidad de la fruta, pero para los primeros estados de desarrollo del cultivo se recomienda la aplicación de fósforo para contribuir a un buen desarrollo de la raíz (Maglianesi, 2013; Gratani, 2014). La fertilización se efectúa mediante varias aplicaciones parciales durante el período de crecimiento; la primera aplicación de nutrientes se realiza al suelo y al follaje aproximadamente dos meses después de la siembra, cuando ya están desarrolladas las raíces, por medios de abonos granulados; sin embargo, la piña, como todas las Bromeliáceas, presenta una absorción foliar más eficaz (Cubero y Sandí, 2013).

Mediante la hibridación, que es un cruce de dos variedades diferentes, se incorporan aquellas características sobresalientes de cada una en la nueva planta, como es el caso del híbrido MD2, que se caracteriza, entre otros aspectos por su madurez temprana (Jiménez, 1999; Valverde, 2004; Aravena, 2005). Esta variedad de piña es de color amarillo, crece sin espinas y sobre todo tiene tolerancia a ciertas plagas y enfermedades, sus flores son de color amarillo con peso promedio de 1.8 a 2 kg por fruto. También es conocida como "Golden Ripe", "Extra Sweet", y "Maya Gold", es un híbrido desarrollado por el Instituto de Investigaciones de Hawai y por la multinacional Del Monte; ha sido muy bien recibida en el mercado europeo, por su buena coloración y sabor, por su presentación y aroma está catalogada como una fruta de lujo en los mercados externos comparada con otras variedades tradicionalmente comercializadas (Pac, 2005). 
La radiación solar, influye en la formación de carbohidratos y consecuentemente en el desarrollo de la planta y su producción; con poca luminosidad el fruto llega a la madurez con aspecto opaco, con adecuada radiación se observa brillante, mientras que elevada intensidad de irradiación sobre el fruto provoca una disminución en su calidad ("quema de sol"), haciéndola inaceptable en mercados fundamentalmente de exportación, la duración óptima de brillo solar es en promedio de 1500 horas por año (Flores et al., 2010).

El suministro de nutrientes a las plantas se puede realizar mediante fertilización granulada al suelo o en forma foliar, es importante conocer la capacidad nutritiva del suelo para aportar los requerimientos del cultivo. A primera cosecha se están recomendado dos fertilizaciones al suelo y catorce foliares, sin embargo en caso de conveniencia se puede aplicar nutrientes foliares mezclados con los fungicidas o insecticidas que se asperjan para el control de plagas y prevención de enfermedades (Fonseca, 2010).

Para cultivar piña se requiere tener en cuenta los siguientes factores ambientales (Flores et al., 2010):

1- Humedad relativa superior al $80 \%$ y temperatura entre 25 y $32^{\circ} \mathrm{C}$

2- Luminosidad adecuada.

3- El suelo debe contener suficiente nitrógeno, $\mathrm{P}_{2} \mathrm{O}_{5}, \mathrm{~K}_{2} \mathrm{O}, \mathrm{CaO}$ y $\mathrm{MgO}$, de no contar con estos minerales, es necesario adicionarlos en plan de fertilización.

4- $\mathrm{pH}$ del suelo alrededor de 5.5 .

5- Los fertilizantes se aplican directamente al suelo y no al fruto.

Existen dos maneras para aplicar los fertirriego, mediante nutrición axilar con bomba de espalda conocida como aplicación directa o por medio del sistema de riego por goteo, ambos métodos tienen experiencias de respaldo que proporcionan confianza a los agricultores, pero no se ha establecido cuál de las dos tiene un mejor rendimiento, por tanto este trabajo se encamino a comparar estos dos métodos de aplicación de fertilizantes, para lo cual fue necesario 
observar el comportamiento de la zona radicular, y otras partes de la planta puesto que su desarrollo está condicionado a la calidad y suministro de nutrientes

\section{METODOLOGÍA}

El presente experimento se realizó en San Martin, Meta, Colombia, que está a una altitud de $420 \mathrm{msnm}$, su temperatura media es 25 a $29^{\circ} \mathrm{C}$, con una humedad relativa promedio de $86 \%$, y la precipitación media anual es 2822 mm (IDEAM, 2016).

La piña variedad MD2 fue sembrada con distancias de $30 \mathrm{~cm}$ entre plantas por 40 cm entre líneas de la cama y un metro entre camas, siguiendo las costumbres y condiciones de la siembra del agricultor de la zona; se realizó evaluación de dos metodologías comparadas con testigo, que fueron considerados como tratamientos, cada uno con una densidad de 600 plantas, T1 = fertilización dirigida al suelo, T2 = fertirriego dirigido a la axila de la planta y T3 = testigo sin ninguna aplicación; los productos fueron aplicados con bomba de espalda y sin boquillas con dirección de acuerdo al método, bien sea en el suelo o en la axila directamente, aclarando que se empleó un producto de fertirriego inicial, con características de disolución totales, lo que permitió manejar dosis exactas (Cuadro 1, Figura 1).

Cuadro1. Cantidad de productos utilizados en los tratamientos por planta

\begin{tabular}{cc}
\hline Días & Fertilización \\
\hline 1 & Dos gr fertirriego mezclado con $0.08 \mathrm{~g}$ de una hormona para \\
& enraizamiento para T1 y T2 \\
30 & Dos g de fertirriego para T1 y T2 \\
45 & Tres g de fertirriego para T1 y T2 \\
60 & Cuatro g de fertirriego para T1 y T2 \\
\hline
\end{tabular}

$\mathrm{T} 1=$ producto dirigido a la axila. $\mathrm{T} 2$ = producto dirigido al suelo T3= Testigo sin ninguna aplicación

Cada repetición estuvo constituida por cinco plantas evaluadas por cama, que constituyó una unidad experimental con medidas de 1 x10 m, en donde había un 
número de 120 plantas, el total del área para cada tratamiento fue de $5 \times 10 \mathrm{~m}$, para un total de 600 plantas por tratamiento; se evaluaron 25 plantas por tratamiento cada 15 días (cinco plantas por cama por repetición); para las medidas de las longitudes se utilizaron reglas y para los pesajes una balanza electrónica, la cual estaba debidamente calibrada.

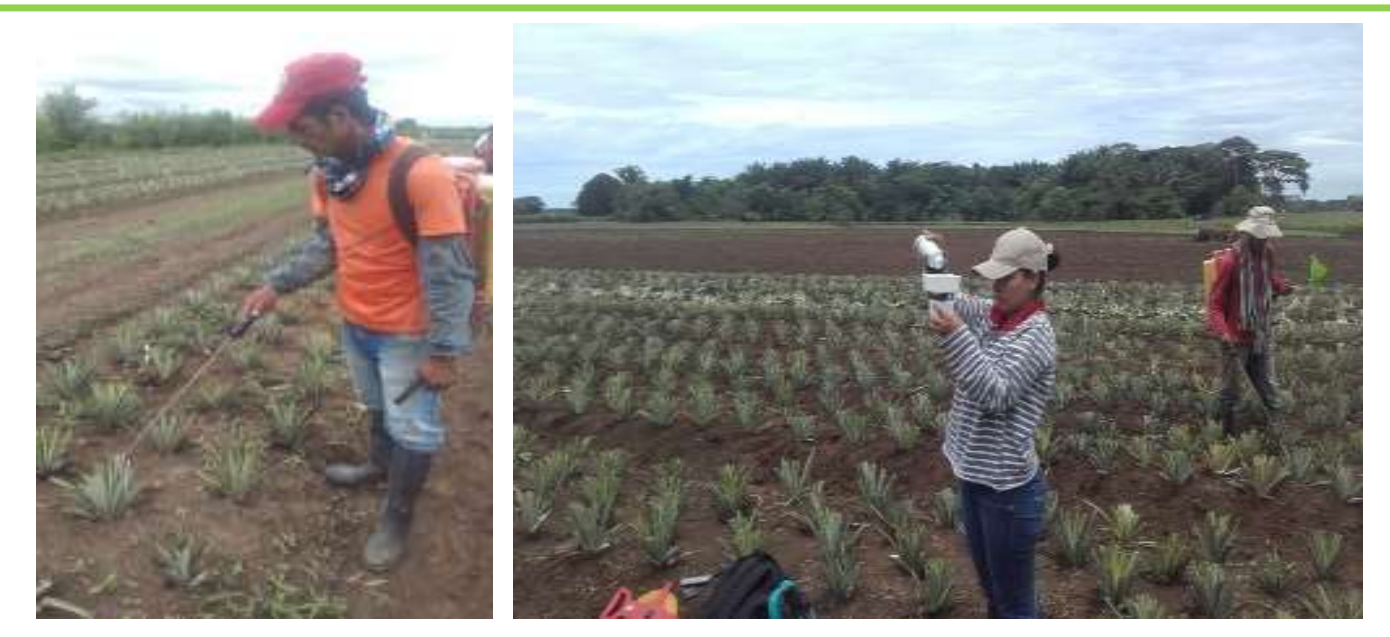

Figura 1. Se utilizaron dosis exactas de fertirriego para aplicar al suelo y a la axila

Este proyecto se estableció bajo un diseño experimental en bloques completos al azar, tres tratamientos, cuatro periodos de muestreo, cinco repeticiones, cinco unidades experimentales, para un total de 300 muestras evaluadas, realizando un análisis de varianza con un nivel de significancia del 5\%, y pruebas de comparación de medias por el método de Duncan.

\section{RESULTADOS Y DISCUSIÓN}

En la primera evaluación de número de hojas no se observó efecto de tratamiento, los valores oscilaron entre 9.60 hojas y 11.40 hojas $(P>0.05)$. En la segunda, tercera y cuarta evaluación (30, 45 y 60 días) T2 presentó los mayores valores en número de hojas $(\mathrm{P}<0.05)$ con $31.80,29.60$ y 33.8 respectivamente en comparación con los otros tratamientos evaluados, aclarando que a los 60 días los valores fueron similares a T1 (Tabla 1). El peso seco de las hojas fue mayor 
$(\mathrm{P}<0.05)$ en todos los cuatro periodos $(15,30,45$ y 60 días) para T2 en comparación con T1 y T3: 18.48, 28.46, 36.78 y 61.67 vs 15.27 y 17.08; 24.0 y $23.99 ; 30.58$ y $27.57 ; 44.5$ y 40.67 respectivamente, siendo a los 60 días superior T2 en 27.8 con respecto T1 y $34.0 \%$ para T3 (Figura 1).

Tabla 1. Efecto de dos tipos de aplicación de fertilizantes en el número de hojas del cultivo de la piña variedad MD2

\begin{tabular}{ccccc}
\hline Tratamientos & 15 Días & 30 Días & 45 Días & 60 Días \\
\hline 1 & $9.60^{\mathrm{a}}$ & $24.60^{\mathrm{a}}$ & $23.40^{\mathrm{a}}$ & $32.20^{\mathrm{ab}}$ \\
2 & $9.60^{\mathrm{a}}$ & $31.80^{\mathrm{b}}$ & $29.60^{\mathrm{b}}$ & $33.80^{\mathrm{b}}$ \\
3 & $11.40^{\mathrm{a}}$ & $22.00^{\mathrm{a}}$ & $21.80^{\mathrm{a}}$ & $27.40^{\mathrm{a}}$ \\
\hline
\end{tabular}

$\mathrm{T} 1=$ producto dirigido a la axila. $\mathrm{T} 2$ = producto dirigido al suelo $\mathrm{T} 3=$ Testigo sin ninguna aplicación. Letras iguales no presentan diferencias significativas $(P>0.05)$

Se puede deducir que a mayor número de hojas la planta va estar mejor debido a que las hojas de la piña tienen una gran actividad fotosintética, y frente a las variaciones del medio ambiente presentan alta plasticidad morfológica y fisiológica, por lo tanto esta parte de la planta sirve para evaluar su crecimiento y estado nutricional, generando inclusive modificaciones fisiológicas, ante situaciones climatológicas adversas, lo cual incide directamente en el crecimiento, desarrollo y productividad de las plantas de piña (Aragón et al., 2012). El concepto conocido como plasticidad fenotípica, comprende los cambios expresados en el fenotipo por un genotipo en diferentes condiciones (Gratani, 2014). Estudios realizados en Bromeliáceas han demostrado que, en términos generales estas especies presentan cierta variabilidad cuando crecen en ambientes contrastantes (Batagin et al., 2009; Cavallero et al., 2011).

El comportamiento de la variable número de raíces, fue similar al de numero de hojas, presentando T2 los mayores valores $(P<0.05)$ a los 30,45 y 60 días (51.4, 81.8 y 90.6 respectivamente) en comparación con T1 (39.20. 69.0 y 75.2) y T3 (33.4, 69.2 y 80.2 respectivamente) (Figura 2). En la evaluación de longitud y peso seco de raíz, a los 15 días no se observó efecto de los tratamientos, mientras que a los 30.45 y 60 días en T2 fueron mayores $(P<0.05)$ en comparación con T1 y T3, siendo a los 60 días superiores estas variables en T2 28.5 y $31.52 \%$ en 
longitud de raíz, y 29.65 y $21.72 \%$ en peso seco de la raíz respectivamente (Tabla 2).

Tabla 2. Efecto de dos tipos de aplicación de fertilizantes en el largo de la raíz (LR) y peso seco de raíz (PSR) del cultivo de la piña variedad MD2

\begin{tabular}{ccccccccc}
\hline & \multicolumn{2}{c}{ 15 Días } & \multicolumn{2}{c}{30 Días } & \multicolumn{2}{c}{ 45 Días } & \multicolumn{2}{c}{60 Días } \\
\cline { 2 - 9 } Tratamiento & $\begin{array}{c}\text { LR } \\
\mathbf{( c m )}\end{array}$ & $\begin{array}{c}\text { PRS } \\
\mathbf{( g )}\end{array}$ & $\begin{array}{c}\text { LR } \\
\mathbf{( c m})\end{array}$ & $\begin{array}{c}\text { PRS } \\
\mathbf{( g )}\end{array}$ & $\begin{array}{c}\text { LR } \\
\mathbf{( c m})\end{array}$ & $\begin{array}{c}\text { PRS } \\
\mathbf{( g )}\end{array}$ & $\begin{array}{c}\text { LR } \\
\mathbf{( c m})\end{array}$ & $\begin{array}{c}\text { PRS } \\
\mathbf{( g )}\end{array}$ \\
\hline T1 & $10.68^{\mathrm{a}}$ & $6.83^{\mathrm{a}}$ & $20.56^{\mathrm{a}}$ & $16.52^{\mathrm{a}}$ & $34.54^{\mathrm{a}}$ & $30.37^{\mathrm{a}}$ & $41.84^{\mathrm{a}}$ & $32.84^{\mathrm{a}}$ \\
T2 & $11.16^{\mathrm{a}}$ & $9.49^{\mathrm{a}}$ & $25.54^{\mathrm{b}}$ & $26.30^{\mathrm{b}}$ & $47.84^{\mathrm{b}}$ & $38.53^{\mathrm{b}}$ & $58.50^{\mathrm{b}}$ & $46.63^{\mathrm{b}}$ \\
T3 & $9.08^{\mathrm{a}}$ & $3.79^{\mathrm{a}}$ & $22.74^{\mathrm{ab}}$ & $13.50^{\mathrm{a}}$ & $29.94^{\mathrm{a}}$ & $27.03^{\mathrm{a}}$ & $40.06^{\mathrm{a}}$ & $36.50^{\mathrm{a}}$ \\
\hline
\end{tabular}

T1 = Producto dirigido a la axila. T2 = Producto dirigido al suelo T3= Testigo sin ninguna aplicación. Letras iguales no presentan diferencias significativas $(P<0.05)$

La función de la raíz es fundamentalmente absorción de agua y sales minerales del suelo por medio de sus pelos absorbentes, hasta la raíz donde son conducidos hacia el tallo y las hojas, donde son transformadas en compuestos orgánicos durante la fotosíntesis. En el interior del tallo y la raíz de la piña se encuentran los meristemos en las yemas situadas en la axila de la hoja (Azcón y Talon, 2000). En la piña se da el brote de raíz por debajo del suelo, siendo sus hojas generalmente más grandes y de mayor peso (Valverde, 2004).

De acuerdo a lo anterior, se puede argumentar que la aplicación del fertirriego en el suelo fue superior, evidenciado en un mejor desarrollo de sistema radicular y foliar, debido a que los nutrimentos son absorbidos con mayor eficiencia por los pelos absorbentes de la raíces que por las axilas, puesto que los meristemos que se encuentran en el interior de la raíz toman con mayor facilidad los nutrientes; mientras que en el fertirriego en las axilas, es posible que la raíz, que es también formadora foliar, no se beneficie de estos compuestos tan importantes para su desarrollo (Figura 2 y Tabla 2).

A los 15 y 30 días de evaluación T1 y T2 presentaron las mayores alturas de la planta $(P<0.05)$ con valores de 15.18 y 43.60 y y 16.24 y $53.20 \mathrm{~cm}$ en comparación con el testigo (T3) 13.93 y 39.44, respectivamente. A los 45 y 60 días T2 mostro 
una altura superior $(\mathrm{P}<0.05)$ con valores de 77.90 y $94.66 \mathrm{~cm}$ con relación a T1 66.52 y $88.30 \mathrm{~cm}$, y T3 68.26 y $79.90 \mathrm{~cm}$ respectivamente; aunque a los 45 días T1 y T2 mostraron un comportamiento similar ( $P>0.05)$ (Figura 3).

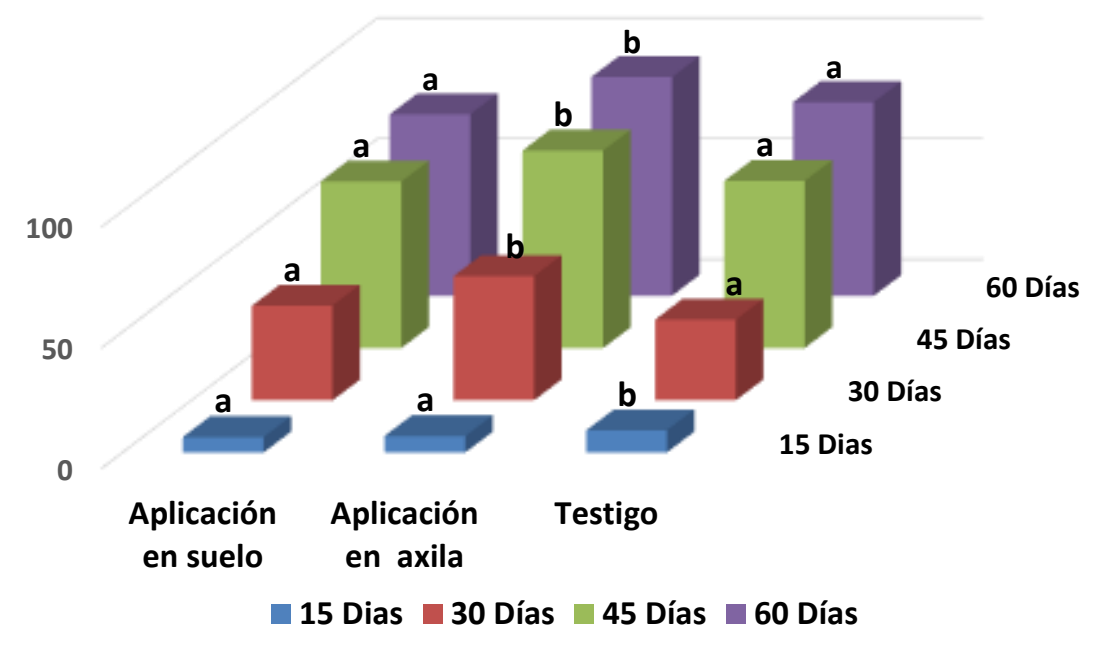

Gráfica 2. Efecto de dos tipos de aplicación de fertilizantes en el número de raíces del cultivo de la piña variedad MD2

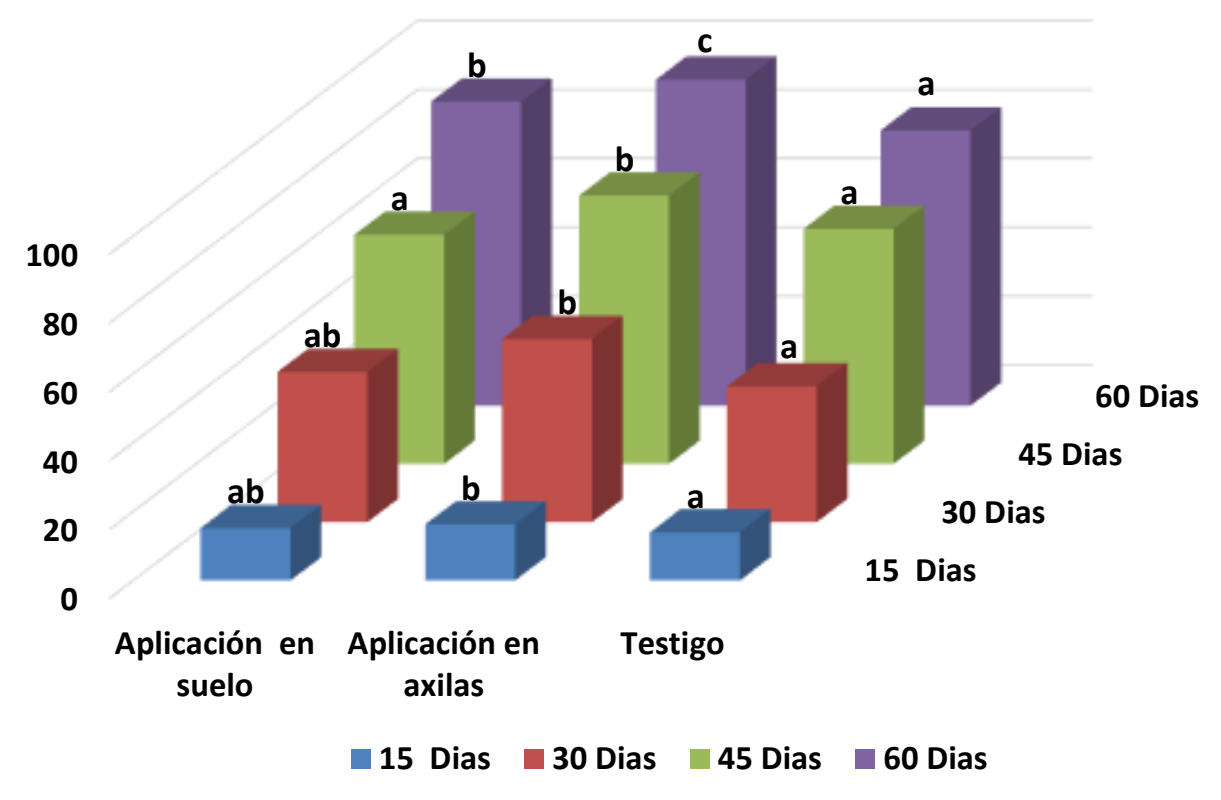

Figura 3. Efecto de dos tipos de aplicación de fertilizantes en altura $(\mathrm{cm})$ del cultivo de la piña variedad MD2 
Las plantas de piña producen un fruto entre los 16 a 24 meses, para lo cual desarrolla un tallo erecto central sobre el que crece el pedúnculo floral que, al madurar origina el fruto múltiple característico. En ese eje central, pedicelos y frutos se unen formando una masa dulce y jugosa, de gran tamaño y sin semillas; la altura de las plantas adultas puede alcanzar 1 a $1.5 \mathrm{~m}$. Bergen, (2005) y Fonseca, (2010) al hacer la comparación con el tamaño del cultivo del experimento, las plantas no alcanzaron el metro de altura, en donde la máxima fue $94.66 \mathrm{~cm}$ con las aplicaciones de fertirriego al suelo, mientras que cuando se realizó en la axila la mayor altura fue $79.9 \mathrm{~cm}$, lo cual difiere a lo hallado en el presente estudio, donde la fertilización aplicada en la axila de la planta presentó el mejor resultado (Figura 3).

\section{CONCLUSIONES}

Según los métodos de fertilización evaluados la aplicación axilar evidenció mejor respuesta por parte de la planta en su sistema radicular y foliar, en comparación con la aplicación directa al suelo y al testigo (sin aplicación de productos), porque las plantas bajo el T1 presentaron características de desarrollo más eficientes para la absorción de nutrientes.

Las plantas del T2 (fertirriego aplicado en axila) presentaron una respuesta fenotípica positiva, evidenciada en número y tamaño de hojas y raíces, manifestándose mejores características fisiológicas relacionadas con el crecimiento y vigor respecto a las plantas de los demás tratamientos

\section{REFERENCIAS BIBLIOGRÁFICAS}

1. Acosta E. La producción piñera en Costa Rica: Un análisis integral de sus implicaciones en el Cantón de Guácimo, Limón, Tesis de Ingeniero Agrónomo con Licenciatura en Ciencias Agrícolas. Escuela de Agricultura de la Región Tropical Húmeda, (EARTH), Guácimo, Limón, Costa Rica. 117 p. 2008.

2. Aragón C., Carvalho L., González J., Escalona M., Amâncio S. The physiology of ex vitro pineapple (Ananas comosus L. Merr. var MD-2) as CAM or C3 is regulated by the environmental conditions. Plant Cell Reports. 31 (4): 757-769. 2012.

3. Aravena J. La expansión piñera en Costa Rica: La realidad de los perdedores de la agroindustria exportadora de la piña. Asociación de Comunidades Ecologistas la Ceiba, San José, Costa Rica. 56 p. 2005. 
4. Azcón J., Talon M. Fundamentos de fisiología vegetal. Ed McGraw-Hill Interamericana, Madrid, España. 522 p. 2000.

5. Batagin K.D., Vieira C., Ossamu F.A., de Almeida M. Alterações morfológicas foliares em piñazeiros cv. IAC Gomo-de-mel micropropagados e aclimatizados em diferentes condições de luminosidade. Acta Botânica Brasílica. 23 (1): 85-92. 2009.

6. Bergen J.A. La expansión piñera en Costa Rica. Asociación de Comunidades Ecologistas la Ceiba, San José, Costa Rica. 56 p. 2005.

7. Cavallero L., Galetti L., López D., McCargo J., Barberis I.M. Morphological variation of the leaves of Aechmea distichantha Lem. plants from contrasting habitats of a Chaco forest: a trade-off between leaf area and mechanical support. Revista Brasileira de Biociencias. 9 (4): 455-464. 2011.

8. Cubero D., Sandí V. Técnicas agroambientales para el manejo del cultivo de piña. Ministerio de Agricultura e Instituto Nacional de Innovación y Transferencia en Tecnología Agropecuaria, San José, Costa Rica. 109 p. 2013.

9. Flores E., Camacho B., Quintero A., Duran D. Presencia de Thielaviopsis paradoxa y Penicillium spp. en frutos de piña en Pampán, estado Trujillo, Venezuela, Comp. Fac. Agron. (LUZ), 2010. Recuperado 07 Jun 2017. Disponible En: http://www.revfacagronluz.org.ve/Compendio\%20Digital/iornadas2010/PDF/fi-21.pdf

10. Fonseca R. Fertilización mediante el método de Stroller en el cultivo de piña (Ananas comosus) (L.) Merr. Híbrido MD-2, en la finca el Tremedal S.A, Tesis Ingeniero Agrónomo. Escuela de Agronomía, Instituto Tecnológico de Costa Rica, San Carlos, Costa Rica. 81 p. 2010.

11. García A., Rodríguez M. Proyecto "Colombia, Costa Rica, Nicaragua: Reduciendo el escurrimiento de plaguicidas al mar Caribe". Manual de Buenas Prácticas Agrícolas para la producción de piña en Costa Rica, BANACOL, Departamento de Asistencia Técnica y Agricultura de, 66 p. 2011. Recuperado 18 Julio 2017. Disponible En: http://cep.unep.org/repcar/proyectos-demostrativos/costa-rica-1/publicacionesbanacol/Manual\%20BPA\%20Banacol.pdf

12. Gratani L. Plant phenotypic plasticity in response to environmental factors. Advances in botany. 2014 (Article ID 208747): 1-17. 2014.

13. IDEAM, Anuario climatológico. Grupo de Climatología y Agro-climatología. Ministerio del medio Ambiente, MINAMBIENTE, Bogotá, Colombia. 356 p. 2016.

14. Jiménez J.A. Manual práctico para el cultivo de piña de exportación. Editorial Tecnológica de Costa Rica, Cartago, Costa Rica. 224 p. 1999.

15. MAG, Ministerio de Agricultura y Ganadería. Manual de buenas prácticas agrícolas para la producción de piña. MAG, Servicio Fitosanitario del Estado, San José, Costa Rica. 136 p. 2010.

16. Maglianesi M.A. Desarrollo de las piñeras en Costa Rica y sus impactos sobre ecosistemas naturales y agro-urbanos. Biocenosis. 27 (1-2): 62-70. 2013.

17. Pac P.J. Experiencias en el cultivo de piña (Annanas comus (L) Merr.) con el hibrido MD2 en la finca La Plata, Coatepeque, Quetzaltenango, Tesis Ingeniero Agrónomo. Instituto de Investigaciones Agronómicas. Facultad de Agronomía, Universidad San Carlos de Guatemala, Guatemala. 48 p. 2005.

18. Valverde R. Comportamiento agronómico del cultivo de piña (Ananas comosus L. Merr) hibrido MD-2 en la localidad del arado, la chorrera, Panamá, Tesis Ingeniero Agrónomo. Instituto Tecnológico de Costa Rica, San Carlos, Costa Rica. 63 p. 2004. 